\title{
Global existence and blow-up analysis for parabolic equations with nonlocal source and nonlinear boundary conditions
}

Wei Kou ${ }^{1}$ and Juntang Ding ${ }^{1 *}$ (D)

"Correspondence:

djuntang@sxu.edu.cn

'School of Mathematical Sciences,

Shanxi University, Taiyuan, P.R. China

\section{Springer}

\begin{abstract}
We investigate the following nonlinear parabolic equations with nonlocal source and nonlinear boundary conditions:

$$
\begin{cases}(g(u))_{t}=\sum_{i, j=1}^{N}\left(a^{i j}(x) u_{x_{i}}\right)_{x_{j}}+\gamma_{1} u^{m}\left(\int_{D} u^{\prime} \mathrm{d} x\right)^{p}-\gamma_{2} u^{r} & \text { in } D \times\left(0, t^{*}\right), \\ \sum_{i, j=1}^{N} a^{i j}(x) u_{x_{i}} v_{j}=h(u) & \text { on } \partial D \times\left(0, t^{*}\right) \\ u(x, 0)=u_{0}(x) \geq 0 & \text { in } \bar{D},\end{cases}
$$

where $p$ and $\gamma_{1}$ are some nonnegative constants, $m, l, \gamma_{2}$, and $r$ are some positive constants, $D \subset \mathbb{R}^{N}(N \geq 2)$ is a bounded convex region with smooth boundary $\partial D$. By making use of differential inequality technique and the embedding theorems in Sobolev spaces and constructing some auxiliary functions, we obtain a criterion to guarantee the global existence of the solution and a criterion to ensure that the solution blows up in finite time. Furthermore, an upper bound and a lower bound for the blow-up time are obtained. Finally, some examples are given to illustrate the results in this paper.
\end{abstract}

MSC: $35 \mathrm{~K} 55 ; 35 B 44$

Keywords: Nonlinear parabolic equations; Blow-up; Nonlocal source; Lower bound

\section{Introduction}

There has been a vast amount of literature to discuss the global solutions, the blow-up solutions, and the bounds for the blow-up time for nonlinear parabolic equations. We refer the readers to [1-16]. The global and blow-up solutions for the parabolic problems with nonlinear boundary conditions have been studied in [1-7]. Recently, some authors have already considered the blow-up phenomena of the parabolic equations with nonlocal source. We recommend the literature [8-10] and the references therein. In the present work, we study the following nonlinear parabolic equations with nonlocal source and non-

(c) The Author(s) 2020. This article is licensed under a Creative Commons Attribution 4.0 International License, which permits use, sharing, adaptation, distribution and reproduction in any medium or format, as long as you give appropriate credit to the original author(s) and the source, provide a link to the Creative Commons licence, and indicate if changes were made. The images or other third party material in this article are included in the article's Creative Commons licence, unless indicated otherwise in a credit line to the material. If material is not included in the article's Creative Commons licence and your intended use is not permitted by statutory regulation or exceeds the permitted use, you will need to obtain permission directly from the copyright holder. To view a copy of this licence, visit http://creativecommons.org/licenses/by/4.0/. 
linear boundary conditions:

$$
\begin{cases}(g(u))_{t}=\sum_{i, j=1}^{N}\left(a^{i j}(x) u_{x_{i}}\right)_{x_{j}}+\gamma_{1} u^{m}\left(\int_{D} u^{l} \mathrm{~d} x\right)^{p}-\gamma_{2} u^{r} & \text { in } D \times\left(0, t^{*}\right), \\ \sum_{i, j=1}^{N} a^{i j}(x) u_{x_{i}} \nu_{j}=h(u) & \text { on } \partial D \times\left(0, t^{*}\right), \\ u(x, 0)=u_{0}(x) \geq 0 & \text { in } \bar{D},\end{cases}
$$

where $p$ and $\gamma_{1}$ are some nonnegative constants, $m, l, \gamma_{2}$, and $r$ are some positive constants, $D \subset \mathbb{R}^{N}(N \geq 2)$ is a bounded convex region, the boundary $\partial D$ is smooth, $t^{*}$ is the blow-up time when blow-up occurs, or $t^{*}=+\infty$, and $v=\left(v_{1}, v_{2}, \ldots, v_{N}\right)$ is the unit outward normal vector on $\partial D$. Moreover, $\left(a^{i j}(x)\right)_{N \times N}$ is a differentiable positive definite matrix; that is, for all $z=\left(z_{1}, z_{2}, \ldots, z_{N}\right) \in \mathbb{R}^{N}$, there exists a constant $\theta>0$ such that

$$
\sum_{i, j=1}^{N} a^{i j} z_{i} z_{j} \geq \theta|z|^{2}
$$

Set $\mathbb{R}_{+}=(0,+\infty)$. We assume that $g$ is a $C^{2}\left(\overline{\mathbb{R}_{+}}\right)$function which satisfies $g^{\prime}(s)>0$ for all $s \in \overline{\mathbb{R}_{+}}, h$ is a nonnegative $C^{1}\left(\overline{\mathbb{R}_{+}}\right)$function, and $u_{0}(x)$ is a nonnegative $C^{1}(\bar{D})$ function which satisfies the compatibility condition. Our study is motivated by the following three papers. Li et al. [1], Baghaei et al. [11], and Zhang et al. [2] studied the following parabolic equations with local source and nonlinear boundary conditions:

$$
\begin{cases}u_{t}=\sum_{i, j=1}^{N}\left(a^{i j}(x) u_{x_{i}}\right)_{x_{j}}+k(t) f(u) & \text { in } D \times\left(0, t^{*}\right) \\ \sum_{i, j=1}^{N} a^{i j}(x) u_{x_{i}} v_{j}=h(u) & \text { on } \partial D \times\left(0, t^{*}\right) \\ u(x, 0)=u_{0}(x) \geq 0 & \text { in } \bar{D},\end{cases}
$$

where $D$ is a bounded convex region in $\mathbb{R}^{N}(N \geq 2)$, and the boundary $\partial D$ is smooth. When the function $k(t) \equiv-1$, Li et al. [1] obtained the conditions to ensure that the global solution exists and the solution blows up in finite time $t^{*}$. Moreover, they derived upper bounds of the blow-up time in $D \subset \mathbb{R}^{N}(N \geq 2)$. Using the three-dimensional Sobolev type inequality that Payne and Schaefer had proven in [7], they got lower bounds in $D \subset \mathbb{R}^{3}$. When $k(t) \equiv-1$, in [11], Baghaei et al. used the embedding theorems in Sobolev spaces to get a lower bound of the blow-up time in $D \subset \mathbb{R}^{N}(N \geq 3)$. Recently, Zhang et al. [2] considered problem (1.3) when $k(t)>0$. Their main contribution was to extend the threedimensional Sobolev type inequality obtained by Payne and Schaefer in [7] to the higher dimensional one. They used this Sobolev inequality in multidimensional space to derive a lower bound of the blow-up time in $D \subset \mathbb{R}^{N}(N \geq 3)$. In addition, they obtained the global existence of the solution and an upper bound of the blow-up time when blow-up occurs in $D \subset \mathbb{R}^{N}(N \geq 2)$.

In the present paper, we study problem (1.1). In the process of getting lower bounds of the blow-up time, the key of our work is to deal with the nonlocal source. We discuss the blow-up problems of (1.1) by constructing some suitable auxiliary functions and making use of the embedding theorems in Sobolev spaces and differential inequality technique. The present work is organized as follows. In Sect. 2, we establish some conditions on the data $g$ and $h$ to obtain that the solution $u(x, t)$ exists globally in $D \subset \mathbb{R}^{N}(N \geq 2)$. In Sect. 3, we develop conditions on the data of (1.1) to guarantee the blow-up of the solution and 
derive an upper bound of blow-up time in $D \subset \mathbb{R}^{N}(N \geq 2)$. When blow-up does occur, we derive a lower bound of $t^{*}$ for $D \subset \mathbb{R}^{N}(N \geq 3)$ in Sect. 4 and a lower bound of $t^{*}$ for $D \subset \mathbb{R}^{2}$ in Sect. 5. In Sect. 6, some examples are presented to illustrate the results in this paper.

\section{The global solution}

In this section, we establish some conditions on the data of (1.1) to ensure that the global solution exists. We define the auxiliary functions

$$
\Phi(t)=\int_{D} G(u) \mathrm{d} x, \quad G(s)=l \int_{0}^{s} y^{l-1} g^{\prime}(y) \mathrm{d} y, \quad s \geq 0 .
$$

Now we state our main results as follows.

Theorem 2.1 Let u be a nonnegative classical solution of problem (1.1). Suppose that the functions $g$ and hatisfy

$$
g^{\prime}(s) \leq \zeta, \quad h(s) \leq \gamma s^{q}, \quad s \geq 0,
$$

where $\zeta, \gamma$, and $q$ are some positive constants. Assume

$$
l>2, \quad q>\max \left\{1, \frac{m+1+l p}{2}\right\}, \quad r>2 q-1 .
$$

Then $u(x, t)$ exists for all $t>0$ in the measure $\Phi(t)$.

Proof It follows from (2.2) that

$$
\Phi(t)=l \int_{D} \int_{0}^{u} y^{l-1} g^{\prime}(y) \mathrm{d} y \mathrm{~d} x \leq \zeta \int_{D} u^{l} \mathrm{~d} x ;
$$

that is,

$$
\int_{D} u^{l} \mathrm{~d} x \geq \frac{1}{\zeta} \Phi(t)
$$

Using (1.2), (2.2), and the divergence theorem, we have

$$
\begin{aligned}
\Phi^{\prime}(t)= & l \int_{D} u^{l-1} g^{\prime}(u) u_{t} \mathrm{~d} x=l \int_{D} u^{l-1}\left(\sum_{i, j=1}^{N}\left(a^{i j}(x) u_{x_{i}}\right)_{x_{j}}+\gamma_{1} u^{m}\left(\int_{D} u^{l} \mathrm{~d} x\right)^{p}-\gamma_{2} u^{r}\right) \mathrm{d} x \\
= & l \int_{D} \sum_{i, j=1}^{N}\left(u^{l-1} a^{i j}(x) u_{x_{i}}\right)_{x_{j}} \mathrm{~d} x-l(l-1) \int_{D} u^{l-2} \sum_{i, j=1}^{N} a^{i j}(x) u_{x_{i}} u_{x_{j}} \mathrm{~d} x \\
& +l \gamma_{1} \int_{D} u^{l+m-1} \mathrm{~d} x\left(\int_{D} u^{l} \mathrm{~d} x\right)^{p}-l \gamma_{2} \int_{D} u^{l+r-1} \mathrm{~d} x \\
= & l \int_{\partial D} u^{l-1} \sum_{i, j=1}^{N} a^{i j}(x) u_{x_{i}} v_{j} \mathrm{~d} S-l(l-1) \int_{D} u^{l-2} \sum_{i, j=1}^{N} a^{i j}(x) u_{x_{i}} u_{x_{j}} \mathrm{~d} x
\end{aligned}
$$




$$
\begin{aligned}
& +l \gamma_{1} \int_{D} u^{l+m-1} \mathrm{~d} x\left(\int_{D} u^{l} \mathrm{~d} x\right)^{p}-l \gamma_{2} \int_{D} u^{l+r-1} \mathrm{~d} x \\
\leq & l \int_{\partial D} u^{l-1} h(u) \mathrm{d} S-\theta l(l-1) \int_{D} u^{l-2}|\nabla u|^{2} \mathrm{~d} x \\
& +l \gamma_{1} \int_{D} u^{l+m-1} \mathrm{~d} x\left(\int_{D} u^{l} \mathrm{~d} x\right)^{p}-l \gamma_{2} \int_{D} u^{l+r-1} \mathrm{~d} x \\
\leq & l \gamma \int_{\partial D} u^{l+q-1} \mathrm{~d} S-\frac{4 \theta(l-1)}{l} \int_{D}\left|\nabla u^{\frac{l}{2}}\right|^{2} \mathrm{~d} x \\
& +l \gamma_{1} \int_{D} u^{l+m-1} \mathrm{~d} x\left(\int_{D} u^{l} \mathrm{~d} x\right)^{p}-l \gamma_{2} \int_{D} u^{l+r-1} \mathrm{~d} x .
\end{aligned}
$$

By the divergence theorem, we get the inequality (see [5])

$$
\int_{\partial D} u^{l+q-1} \mathrm{~d} S \leq \frac{N}{\rho_{0}} \int_{D} u^{l+q-1} \mathrm{~d} x+\frac{(l+q-1) d}{\rho_{0}} \int_{D} u^{l+q-2}|\nabla u| \mathrm{d} x
$$

where

$$
\rho_{0}=\min _{x \in \partial D}(x \cdot v), \quad d=\max _{x \in \bar{D}}|x| .
$$

Applying the Hölder inequality and the Young inequality to the right-hand side of (2.6) yields

$$
\begin{aligned}
\frac{N}{\rho_{0}} \int_{D} u^{l+q-1} \mathrm{~d} x & \leq\left(\int_{D}\left(u^{\frac{l}{2}}\right)^{2} \mathrm{~d} x\right)^{\frac{1}{2}}\left(\int_{D}\left(\frac{N}{\rho_{0}} u^{\frac{l+2 q-2}{2}}\right)^{2} \mathrm{~d} x\right)^{\frac{1}{2}} \\
& \leq \frac{1}{2} \int_{D} u^{l} \mathrm{~d} x+\frac{N^{2}}{2 \rho_{0}^{2}} \int_{D} u^{l+2 q-2} \mathrm{~d} x
\end{aligned}
$$

and

$$
\begin{aligned}
& \frac{(l+q-1) d}{\rho_{0}} \int_{D} u^{l+q-2}|\nabla u| \mathrm{d} x \\
& \leq\left(\int_{D} \frac{1}{\varepsilon}\left(\frac{(l+q-1) d}{\rho_{0}} u^{\frac{l+2 q-2}{2}}\right)^{2} \mathrm{~d} x\right)^{\frac{1}{2}}\left(\int_{D} \varepsilon\left(u^{\frac{l-2}{2}}|\nabla u|\right)^{2} \mathrm{~d} x\right)^{\frac{1}{2}} \\
& \leq \frac{(l+q-1)^{2} d^{2}}{2 \varepsilon \rho_{0}^{2}} \int_{D} u^{l+2 q-2} \mathrm{~d} x+\frac{\varepsilon}{2} \int_{D} u^{l-2}|\nabla u|^{2} \mathrm{~d} x \\
& =\frac{(l+q-1)^{2} d^{2}}{2 \varepsilon \rho_{0}^{2}} \int_{D} u^{l+2 q-2} \mathrm{~d} x+\frac{2 \varepsilon}{l^{2}} \int_{D}\left|\nabla u^{\frac{l}{2}}\right|^{2} \mathrm{~d} x
\end{aligned}
$$

with

$$
\varepsilon=\frac{2 \theta(l-1)}{\gamma}>0
$$


Hence, substituting (2.7) and (2.8) into (2.6), we get

$$
\begin{aligned}
\int_{\partial D} u^{l+q-1} \mathrm{~d} S \leq & \frac{1}{2} \int_{D} u^{l} \mathrm{~d} x+\left(\frac{N^{2}}{2 \rho_{0}^{2}}+\frac{(l+q-1)^{2} d^{2}}{2 \varepsilon \rho_{0}^{2}}\right) \int_{D} u^{l+2 q-2} \mathrm{~d} x \\
& +\frac{2 \varepsilon}{l^{2}} \int_{D}\left|\nabla u^{\frac{l}{2}}\right|^{2} \mathrm{~d} x .
\end{aligned}
$$

Inserting (2.10) into (2.5) and using (2.9), we obtain

$$
\begin{aligned}
\Phi^{\prime}(t) \leq & \frac{1}{2} l \gamma \int_{D} u^{l} \mathrm{~d} x+l \gamma\left(\frac{N^{2}}{2 \rho_{0}^{2}}+\frac{(l+q-1)^{2} d^{2}}{2 \varepsilon \rho_{0}^{2}}\right) \int_{D} u^{l+2 q-2} \mathrm{~d} x \\
& +l \gamma_{1} \int_{D} u^{l+m-1} \mathrm{~d} x\left(\int_{D} u^{l} \mathrm{~d} x\right)^{p}-l \gamma_{2} \int_{D} u^{l+r-1} \mathrm{~d} x .
\end{aligned}
$$

Since (2.3) implies the fact that $0<\frac{l}{l+2 q-2}<1,0<\frac{l+m-1}{l+2 q-2}<1$, and $0<\frac{l+2 q-2}{l+s-1}<1$, it follows from the Hölder inequality that

$$
\begin{aligned}
& \int_{D} u^{l} \mathrm{~d} x \leq|D|^{\frac{2(q-1)}{l+2 q-2}}\left(\int_{D} u^{l+2 q-2} \mathrm{~d} x\right)^{\frac{l}{l+2 q-2}}, \\
& \int_{D} u^{l+m-1} \mathrm{~d} x \leq|D|^{\frac{2 q-m-1}{l+2 q-2}}\left(\int_{D} u^{l+2 q-2} \mathrm{~d} x\right)^{\frac{l+m-1}{l+2 q-2}},
\end{aligned}
$$

and

$$
\int_{D} u^{l+2 q-2} \mathrm{~d} x \leq|D|^{\frac{r-2 q+1}{l+r-1}}\left(\int_{D} u^{l+r-1} \mathrm{~d} x\right)^{\frac{l+2 q-2}{l+r-1}},
$$

where $|D|$ is the volume of $D$. By virtue of (2.14), we have

$$
\int_{D} u^{l+r-1} \mathrm{~d} x \geq|D|^{\frac{2 q-r-1}{l+2 q-2}}\left(\int_{D} u^{l+2 q-2} \mathrm{~d} x\right)^{\frac{l+r-1}{l+2 q-2}} .
$$

We insert (2.12)-(2.13) and (2.15) into (2.11) to derive

$$
\begin{aligned}
\Phi^{\prime}(t) \leq & \frac{1}{2} l \gamma|D|^{\frac{2(q-1)}{l+2 q-2}}\left(\int_{D} u^{l+2 q-2} \mathrm{~d} x\right)^{\frac{l}{l+2 q-2}}+l \gamma\left(\frac{N^{2}}{2 \rho_{0}^{2}}+\frac{(l+q-1)^{2} d^{2}}{2 \varepsilon \rho_{0}^{2}}\right) \int_{D} u^{l+2 q-2} \mathrm{~d} x \\
& +l \gamma_{1}|D|^{\frac{2 q-m-1+2 p(q-1)}{l+2 q-2}}\left(\int_{D} u^{l+2 q-2} \mathrm{~d} x\right)^{\frac{l+m-1+l p}{l+2 q-2}} \\
& -l \gamma_{2}|D|^{\frac{2 q-r-1}{l+2 q-2}}\left(\int_{D} u^{l+2 q-2} \mathrm{~d} x\right)^{\frac{l+r-1}{l+2 q-2}} \\
= & \int_{\Omega} u^{l+2 q-2} \mathrm{~d} x\left(l \gamma\left(\frac{N^{2}}{2 \rho_{0}^{2}}+\frac{(l+q-1)^{2} d^{2}}{2 \varepsilon \rho_{0}^{2}}\right)+\frac{l \gamma}{2}|D|^{\frac{2(q-1)}{l+2 q-2}}\left(\int_{D} u^{l+2 q-2} \mathrm{~d} x\right)^{\frac{2(1-q)}{l+2 q-2}}\right. \\
& +l \gamma_{1}|D|^{\frac{2 q-m-1+2 p(q-1)}{l+2 q-2}}\left(\int_{D} u^{l+2 q-2} \mathrm{~d} x\right)^{\frac{m-2 q+1+l p}{l+2 q-2}} \\
& \left.-l \gamma_{2}|D|^{\frac{2 q-r-1}{l+2 q-2}}\left(\int_{D} u^{l+2 q-2} \mathrm{~d} x\right)^{\frac{r-2 q+1}{l+2 q-2}}\right) .
\end{aligned}
$$


It follows from (2.12) and (2.4) that

$$
\int_{D} u^{l+2 q-2} \mathrm{~d} x \geq|D|^{\frac{2(1-q)}{l}}\left(\int_{D} u^{l} \mathrm{~d} x\right)^{\frac{l+2 q-2}{l}} \geq \zeta^{\frac{2-2 q-l}{l}}|D|^{\frac{2(1-q)}{l}} \Phi^{\frac{l+2 q-2}{l}}(t)
$$

By (2.3), we know $\frac{2(1-q)}{l+2 q-2}<0, \frac{m-2 q+1+l p}{l+2 q-2}<0$, and $\frac{r-2 q+1}{l+2 q-2}>0$. Substituting (2.17) into (2.16), we deduce

$$
\begin{aligned}
\Phi^{\prime}(t) \leq & \int_{D} u^{l+2 q-2} \mathrm{~d} x\left[l \gamma\left(\frac{N^{2}}{2 \rho_{0}^{2}}+\frac{(l+q-1)^{2} d^{2}}{2 \varepsilon \rho_{0}^{2}}\right)+\frac{l \gamma}{2} \zeta^{\frac{2(q-1)}{l}}|D|^{\frac{2(q-1)}{l}} \Phi^{\frac{2(1-q)}{l}}(t)\right. \\
& \left.+l \gamma_{1} \zeta^{\frac{2 q-m-1}{l}-p}|D|^{\frac{2 q-m-1}{l}} \Phi^{p+\frac{m-2 q+1}{l}}(t)-l \gamma_{2} \zeta^{\frac{2 q-r-1}{l}}|D|^{\frac{2 q-r-1}{l}} \Phi^{\frac{r-2 q+1}{l}}(t)\right] \\
= & \int_{D} u^{l+2 q-2} \mathrm{~d} x\left(C_{1}+C_{2} \Phi^{\frac{2(1-q)}{l}}(t)+C_{3} \Phi^{p+\frac{m-2 q+1}{l}}(t)-C_{4} \Phi^{\frac{r-2 q+1}{l}}(t)\right)
\end{aligned}
$$

where $C_{1}=l \gamma\left(\frac{N^{2}}{2 \rho_{0}^{2}}+\frac{(l+q-1)^{2} d^{2}}{2 \varepsilon \rho_{0}^{2}}\right), C_{2}=\frac{l \gamma}{2} \zeta^{\frac{2(q-1)}{l}}|D|^{\frac{2(q-1)}{l}}, C_{3}=l \gamma_{1} \zeta^{\frac{2 q-m-1}{l}-p}|D|^{\frac{2 q-m-1}{l}}$, and $C_{4}=$ $l \gamma_{2} \zeta^{\frac{2 q-r-1}{l}}|D|^{\frac{2 q-r-1}{l}}$.

Thus, (2.18) implies that $u(t)$ cannot blow up for all time $t>0$ in the measure $\Phi(t)$. In fact, if $u(x, t)$ blows up at finite time $t^{*}$ in the measure $\Phi(t)$, we have

$$
\lim _{t \rightarrow t^{*-}} \Phi(t)=+\infty
$$

It follows from (2.3) that $\frac{2-2 q}{l}<0, p+\frac{m-2 q+1}{l}<0$, and $\frac{r-2 q+1}{l}>0$. From (2.18), we obtain that $\Phi^{\prime}(t)<0$ in some interval $\left(t_{0}, t^{*}\right)$. So, for any $t \in\left[t_{0}, t^{*}\right)$, we get $\Phi(t) \leq \Phi\left(t_{0}\right)$. We take the limit as $t \rightarrow t^{*-}$ to get

$$
+\infty=\lim _{t \rightarrow t^{*-}} \Phi(t) \leq \Phi\left(t_{0}\right)
$$

This is a contradiction.

\section{Upper bounds of the blow-up time $t^{*}$}

In this section, we set up some conditions on $g$ and $k$ to guarantee that the solution of (1.1) blows up in finite time. An upper bound of the blow-up time $t^{*}$ is obtained in $D \subset \mathbb{R}^{N}$ $(N \geq 2)$. The auxiliary functions of this section are defined as follows:

$$
\Psi(t)=\int_{D} g(u) \mathrm{d} x, \quad t \geq 0
$$

Our main result is the following Theorem 3.1.

Theorem 3.1 Let $u(x, t)$ be a nonnegative classical solution of problem (1.1). Suppose that the function $g$ satisfies

$$
0 \leq g(s) \leq s, \quad s \geq 0
$$

Assume

$$
1<r<\min \{m, l\} \text { and } \Psi(0)>\left(\frac{M_{2}}{M_{1}}\right)^{\frac{1}{m+l p}}
$$


with

$$
\begin{aligned}
& M_{1}=\frac{\gamma_{1}}{2}|D|^{1+p-m-l p}, \quad M_{2}=\frac{\gamma_{2}(m+l p-r)}{m+l p} \delta^{\frac{r}{r-m-l p}}, \\
& \delta=\frac{\gamma_{1}(m+l p)}{2 r \gamma_{2}}|D|^{1+p-\frac{m+l p}{r}} .
\end{aligned}
$$

Then the solution $u(x, t)$ must blow up in the measure $\Psi(t)$ in finite time $t^{*}$ and

$$
t^{*} \leq \int_{\Psi(0)}^{+\infty} \frac{d \tau}{M_{1} \tau^{m+l p}-M_{2}} .
$$

Proof Making use of the divergence theorem, we get

$$
\begin{aligned}
\Psi^{\prime}(t) & =\int_{D} g^{\prime}(u) u_{t} \mathrm{~d} x=\int_{D}\left(\sum_{i, j=1}^{N}\left(a^{i j}(x) u_{x_{i}}\right)_{x_{j}}+\gamma_{1} u^{m}\left(\int_{D} u^{l} \mathrm{~d} x\right)^{p}-\gamma_{2} u^{r}\right) \mathrm{d} x \\
& =\int_{\partial D} \sum_{i, j=1}^{N} a^{i j}(x) u_{x_{i}} v_{j} \mathrm{~d} S+\gamma_{1} \int_{D} u^{m} \mathrm{~d} x\left(\int_{D} u^{l} \mathrm{~d} x\right)^{p}-\gamma_{2} \int_{D} u^{r} \mathrm{~d} x \\
& =\int_{\partial D} h(u) \mathrm{d} S+\gamma_{1} \int_{D} u^{m} \mathrm{~d} x\left(\int_{D} u^{l} \mathrm{~d} x\right)^{p}-\gamma_{2} \int_{D} u^{r} \mathrm{~d} x \\
& \geq \gamma_{1} \int_{D} u^{m} \mathrm{~d} x\left(\int_{D} u^{l} \mathrm{~d} x\right)^{p}-\gamma_{2} \int_{D} u^{r} \mathrm{~d} x .
\end{aligned}
$$

By the Hölder inequality and (3.3), we can compute

$$
\begin{aligned}
& \int_{D} u^{r} \mathrm{~d} x \leq|D|^{1-\frac{r}{m}}\left(\int_{D} u^{m} \mathrm{~d} x\right)^{\frac{r}{m}}, \quad \int_{D} u^{r} \mathrm{~d} x \leq|D|^{1-\frac{r}{l}}\left(\int_{D} u^{l} \mathrm{~d} x\right)^{\frac{r}{l}}, \\
& \int_{D} u \mathrm{~d} x \leq|D|^{1-\frac{1}{r}}\left(\int_{D} u^{r} \mathrm{~d} x\right)^{\frac{1}{r}} ;
\end{aligned}
$$

that is,

$$
\begin{aligned}
& \int_{D} u^{m} \mathrm{~d} x \geq|D|^{1-\frac{m}{r}}\left(\int_{D} u^{r} \mathrm{~d} x\right)^{\frac{m}{r}}, \quad \int_{D} u^{l} \mathrm{~d} x \geq|D|^{1-\frac{l}{r}}\left(\int_{D} u^{r} \mathrm{~d} x\right)^{\frac{l}{r}}, \\
& \int_{D} u^{r} \mathrm{~d} x \geq|D|^{1-r}\left(\int_{D} u \mathrm{~d} x\right)^{r} .
\end{aligned}
$$

Substituting (3.6) into (3.5) and applying the Young inequality and (3.2)-(3.4), we deduce

$$
\begin{aligned}
\Psi^{\prime}(t) & \geq \gamma_{1}|D|^{1+p-\frac{m+l p}{r}}\left(\int_{D} u^{r} \mathrm{~d} x\right)^{\frac{m+l p}{r}}-\gamma_{2} \int_{D} u^{r} \mathrm{~d} x \\
& =\gamma_{1}|D|^{1+p-\frac{m+l p}{r}}\left(\int_{D} u^{r} \mathrm{~d} x\right)^{\frac{m+l p}{r}}-\gamma_{2}\left(\delta\left(\int_{D} u^{r} \mathrm{~d} x\right)^{\frac{m+l p}{r}}\right)^{\frac{r}{m+l p}}\left(\delta^{\left.\frac{r}{r-m-l p}\right)^{\frac{m+l p-r}{m+l p}}}\right. \\
& \geq\left(\gamma_{1}|D|^{1+p-\frac{m+l p}{r}}-\frac{\delta r \gamma_{2}}{m+l p}\right)\left(\int_{D} u^{r} \mathrm{~d} x\right)^{\frac{m+l p}{r}}-\frac{\gamma_{2}(m+l p-r)}{m+l p} \delta^{\frac{r}{r-m-l p}}
\end{aligned}
$$




$$
\begin{aligned}
& \geq\left(\gamma_{1}|D|^{1+p-\frac{m+l p}{r}}-\frac{\delta r \gamma_{2}}{m+l p}\right)|D|^{\frac{(1-r)(m+l p)}{r}}\left(\int_{D} u \mathrm{~d} x\right)^{m+l p}-\frac{\gamma_{2}(m+l p-r)}{m+l p} \delta^{\frac{r}{r-m-l p}} \\
& =M_{1}\left(\int_{D} u \mathrm{~d} x\right)^{m+l p}-M_{2} \geq M_{1}\left(\int_{D} g(u) \mathrm{d} x\right)^{m+l p}-M_{2} \\
& =M_{1} \Psi^{m+l p}(t)-M_{2}
\end{aligned}
$$

We know that (3.3) implies

$$
M_{1} \Psi^{m+l p}(t)-M_{2}>0, \quad t \geq 0
$$

In fact, if (3.8) does not hold, we let

$$
t^{\prime}=\min \left\{M_{1} \Psi^{m+l p}(t)-M_{2} \leq 0\right\} .
$$

From (3.9), we have

$$
M_{1} \Psi^{m+l p}(t)-M_{2}>0, \quad 0<t<t^{\prime}
$$

It follows from (3.7) and (3.10) that $\Psi^{\prime}(t)>0,0<t<t^{\prime}$, and $\Psi\left(t^{\prime}\right)>\Psi(0)$. By (3.3), we derive

$$
M_{1} \Psi^{m+l p}\left(t^{\prime}\right)-M_{2}>0
$$

which contradicts with (3.9).

Integrating (3.7) over $[0, t]$, we obtain

$$
t \leq \int_{\Psi(0)}^{\Psi(t)} \frac{d \tau}{M_{1} \tau^{m+l p}-M_{2}}
$$

which implies that the solution $u$ must blow up at some finite time $t^{*}$ in the measure $\Psi(t)$. In fact, if $u$ does not blow up at $t^{*}$ in the measure $\Psi(t)$, we get

$$
\Psi(t)<+\infty, \quad t \geq 0 .
$$

Therefore,

$$
t \leq \int_{\Psi(0)}^{\Psi(t)} \frac{d \tau}{M_{1} \tau^{m+l p}-M_{2}}<\int_{\Psi(0)}^{+\infty} \frac{d \tau}{M_{1} \tau^{m+l p}-M_{2}}, \quad t \geq 0 .
$$

Letting $t \rightarrow+\infty$, we have

$$
\int_{\Psi(0)}^{+\infty} \frac{d \tau}{M_{1} \tau^{m+l p}-M_{2}}=+\infty
$$

which contradicts with

$$
\int_{\Psi(0)}^{+\infty} \frac{d \tau}{M_{1} \tau^{m+l p}-M_{2}}<+\infty
$$


Hence, $u$ blows up at $t^{*}$ in the measure $\Psi(t)$. We pass to the limit as $t \rightarrow t^{*-}$ in (3.11) to derive

$$
t^{*} \leq \int_{\Psi(0)}^{+\infty} \frac{d \tau}{M_{1} \tau^{m+l p}-M_{2}}
$$

4 Lower bounds of the blow-up time $t^{*}$ in $D \subset \mathbb{R}^{N}(N \geq 3)$

In this section, we impose restriction $D \subset \mathbb{R}^{N}(N \geq 3)$. Assume that the functions $h$ and $g$ satisfy

$$
h(s) \leq \sigma s^{q}, \quad g^{\prime}(s) \geq \xi, \quad s \geq 0,
$$

where $\sigma, q$, and $\xi$ are some positive constants and

$$
q>1 \text {. }
$$

It follows from [17, Corollary 9.14] that

$$
W^{1,2} \hookrightarrow L^{\frac{2 N}{N-2}}, \quad \text { where } N \geq 3,
$$

from which we have the following Sobolev inequality:

$$
\left(\int_{D}\left(u^{\frac{l}{2}}\right)^{\frac{2 N}{N-2}} \mathrm{~d} x\right)^{\frac{N-2}{2 N}} \leq C\left(\int_{D} u^{l} \mathrm{~d} x+\int_{D}\left|\nabla u^{\frac{l}{2}}\right|^{2} \mathrm{~d} x\right)^{\frac{1}{2}}
$$

where $C=C(N, D)$ is a Sobolev embedding constant depending on $N(N \geq 3)$ and $D$. In order to derive a lower bound of the blow-up time $t^{*}$, we use inequality (4.3) and

$$
(a+b)^{\mu} \leq a^{\mu}+b^{\mu}, \quad a \geq 0, b \geq 0,0<\mu \leq 1
$$

We define auxiliary functions as follows:

$$
A(t)=\int_{D} B(u) \mathrm{d} x, \quad t \geq 0, \quad B(s)=l \int_{0}^{s} y^{l-1} g^{\prime}(y) \mathrm{d} y, \quad s \geq 0 .
$$

Now we present our main results in Theorem 4.1.

Theorem 4.1 Let $u(x, t)$ be a nonnegative classical solution of $(1.1)$ in $D \subset \mathbb{R}^{N}(N \geq 3)$. Suppose that (4.1)-(4.2) hold and

$$
r>1, \quad m>1, \quad l>\max \left\{2, \frac{N(m-1)}{2}, N(q-1)\right\} .
$$

If $u(x, t)$ becomes unbounded at some finite time $t^{*}$ in the measure $A(t)$, then we conclude $t^{*}$ is bounded from below by

$$
t^{*} \geq \int_{A(0)}^{+\infty} \frac{\mathrm{d} \tau}{J_{1} \tau+J_{2} \tau^{1+\frac{2(q-1)}{l}}+J_{3} \tau^{1+\frac{2(q-1)}{l-N(q-1)}}+J_{4} \tau^{\frac{(l p+m-1)(l+r-1)}{l(r-1)}}+J_{5} \tau^{1+\frac{2 p l+2(m-1)}{2 l-N(m-1)}}},
$$


where

$$
\begin{aligned}
& J_{1}=\frac{l \sigma}{2 \xi}, \quad J_{2}=\frac{l \sigma}{2 \rho_{0}^{2} \xi^{1+\frac{2(q-1)}{l}}} C^{\frac{2 N(q-1)}{l}}\left(N^{2}+\frac{\sigma(l+q-1)^{2} d^{2}}{\theta(l-1)}\right) \\
& J_{3}=\frac{l-N(q-1)}{\xi^{1+\frac{2(q-1)}{l-N(q-1)}}}\left(\frac{\theta(l-1)}{l N(q-1) C^{2}}\right)^{\frac{N(1-q)}{l-N(q-1)}}\left[\frac{\sigma}{2 \rho_{0}^{2}}\left(N^{2}+\frac{\sigma(l+q-1)^{2} d^{2}}{\theta(l-1)}\right)\right]^{\frac{l}{l-N(q-1)}}, \\
& J_{4}=\frac{|D|(r-1)}{\xi^{\frac{(l p+m-1)(l+r-1)}{l(r-1)}}} \gamma_{2}^{\frac{l}{1-r}}\left(\frac{l \gamma_{1}}{l+r-1} C^{\frac{N(m-1)}{l}}\right)^{1+\frac{l}{r-1}}, \\
& J_{5}=\frac{2 l-N(m-1)}{\xi^{1+\frac{2 p l+2(m-1)}{2 l-N(m-1)}}}\left(\frac{\gamma_{1}}{2}\right)^{\frac{2 l}{2 l-N(m-1)}}\left(\frac{\theta(l-1)}{l N(m-1) C^{2}}\right)^{\frac{N(1-m)}{2 l-N(m-1)}}, \\
& \rho_{0}=\min _{x \in \partial D}(x \cdot v), \quad d=\max _{x \in \bar{D}}|x|,
\end{aligned}
$$

and $\theta$ is defined in (1.2).

Proof We use (1.2), (4.1), and the divergence theorem to obtain

$$
\begin{aligned}
A^{\prime}(t)= & l \int_{D} u^{l-1} g^{\prime}(u) u_{t} \mathrm{~d} x=l \int_{D} u^{l-1}\left(\sum_{i, j=1}^{N}\left(a^{i j}(x) u_{x_{i}}\right)_{x_{j}}+\gamma_{1} u^{m}\left(\int_{D} u^{l} \mathrm{~d} x\right)^{p}-\gamma_{2} u^{r}\right) \mathrm{d} x \\
= & l \int_{D} \sum_{i, j=1}^{N}\left(u^{l-1} a^{i j}(x) u_{x_{i}}\right)_{x_{j}} \mathrm{~d} x-l(l-1) \int_{D} u^{l-2} \sum_{i, j=1}^{N} a^{i j}(x) u_{x_{i}} u_{x_{j}} \mathrm{~d} x \\
& +l \gamma_{1} \int_{D} u^{l+m-1} \mathrm{~d} x\left(\int_{D} u^{l} \mathrm{~d} x\right)^{p}-l \gamma_{2} \int_{D} u^{l+r-1} \mathrm{~d} x \\
\leq & l \int_{\partial D} u^{l-1} \sum_{i, j=1}^{N} a^{i j}(x) u_{x_{i}} v_{j} \mathrm{~d} S-l \theta(l-1) \int_{D} u^{l-2}|\nabla u|^{2} \mathrm{~d} x \\
& +l \gamma_{1} \int_{D} u^{l+m-1} \mathrm{~d} x\left(\int_{D} u^{l} \mathrm{~d} x\right)^{p}-l \gamma_{2} \int_{D} u^{l+r-1} \mathrm{~d} x \\
\leq & l \int_{\partial D} u^{l-1} h(u) \mathrm{d} S-\frac{4 \theta(l-1)}{l} \int_{D}\left|\nabla u^{\frac{l}{2}}\right|^{2} \mathrm{~d} x \\
& +l \gamma_{1} \int_{D} u^{l+m-1} \mathrm{~d} x\left(\int_{D} u^{l} \mathrm{~d} x\right)^{p}-l \gamma_{2} \int_{D} u^{l+r-1} \mathrm{~d} x \\
\leq & l \sigma \int_{\partial D} u^{l+q-1} \mathrm{~d} S-\frac{4 \theta(l-1)}{l} \int_{D}\left|\nabla u^{\frac{l}{2}}\right|^{2} \mathrm{~d} x \\
& +l \gamma_{1} \int_{D} u^{l+m-1} \mathrm{~d} x\left(\int_{D} u^{l} \mathrm{~d} x\right)^{p}-l \gamma_{2} \int_{D} u^{l+r-1} \mathrm{~d} x
\end{aligned}
$$

Repeating the calculation process of (2.6)-(2.8), we have

$$
\begin{aligned}
\int_{\partial D} u^{l+q-1} \mathrm{~d} S \leq & \frac{1}{2} \int_{D} u^{l} \mathrm{~d} x+\left(\frac{N^{2}}{2 \rho_{0}^{2}}+\frac{(l+q-1)^{2} d^{2}}{2 \varepsilon_{1} \rho_{0}^{2}}\right) \int_{D} u^{l+2 q-2} \mathrm{~d} x \\
& +\frac{2 \varepsilon_{1}}{l^{2}} \int_{D}\left|\nabla u^{\frac{l}{2}}\right|^{2} \mathrm{~d} x,
\end{aligned}
$$


where $\rho_{0}$ and $d$ are given in (4.11) and $\varepsilon_{1}=\frac{\theta(l-1)}{\sigma}$. Substituting (4.13) into (4.12), we derive

$$
\begin{aligned}
A^{\prime}(t) \leq & \frac{l \sigma}{2} \int_{D} u^{l} \mathrm{~d} x+l \sigma\left(\frac{N^{2}}{2 \rho_{0}^{2}}+\frac{(l+q-1)^{2} d^{2}}{2 \varepsilon_{1} \rho_{0}^{2}}\right) \int_{D} u^{l+2 q-2} \mathrm{~d} x \\
& +\left(\frac{2 \varepsilon_{1} \sigma}{l}-\frac{4 \theta(l-1)}{l}\right) \int_{D}\left|\nabla u^{\frac{l}{2}}\right|^{2} \mathrm{~d} x \\
& +l \gamma_{1} \int_{D} u^{l+m-1} \mathrm{~d} x\left(\int_{D} u^{l} \mathrm{~d} x\right)^{p}-l \gamma_{2} \int_{D} u^{l+r-1} \mathrm{~d} x .
\end{aligned}
$$

First we take into account the second term of (4.14). Assumptions (4.2) and (4.6) imply $0<\frac{(N-2)(q-1)}{l}<1$ and $0<\frac{N(q-1)}{l}<1$. Now we apply the Hölder inequality, the Young inequality, and inequalities (4.3)-(4.4) to deduce

$$
\begin{aligned}
& \int_{D} u^{l+2 q-2} \mathrm{~d} x \\
& \leq\left(\int_{D} u^{l} \mathrm{~d} x\right)^{1-\frac{(N-2)(q-1)}{l}}\left(\int_{D}\left(u^{\frac{l}{2}}\right)^{\frac{2 N}{N-2}} \mathrm{~d} x\right)^{\frac{(N-2)(q-1)}{l}} \\
& =\left(\int_{D} u^{l} \mathrm{~d} x\right)^{1-\frac{(N-2)(q-1)}{l}}\left(\left(\int_{D}\left(u^{\frac{l}{2}}\right)^{\frac{2 N}{N-2}} \mathrm{~d} x\right)^{\frac{N-2}{2 N}}\right)^{\frac{2 N(q-1)}{l}} \\
& \leq\left(\int_{D} u^{l} \mathrm{~d} x\right)^{1-\frac{(N-2)(q-1)}{l}}\left(C\left(\int_{D} u^{l} \mathrm{~d} x+\int_{D}\left|\nabla u^{\frac{l}{2}}\right|^{2} \mathrm{~d} x\right)^{\frac{1}{2}}\right)^{\frac{2 N(q-1)}{l}} \\
& =C^{\frac{2 N(q-1)}{l}}\left(\int_{D} u^{l} \mathrm{~d} x\right)^{1-\frac{(N-2)(q-1)}{l}}\left(\int_{D} u^{l} \mathrm{~d} x+\int_{D}\left|\nabla u^{\frac{l}{2}}\right|^{2} \mathrm{~d} x\right)^{\frac{N(q-1)}{l}} \\
& \leq C^{\frac{2 N(q-1)}{l}}\left(\int_{D} u^{l} \mathrm{~d} x\right)^{1-\frac{(N-2)(q-1)}{l}}\left(\left(\int_{D} u^{l} \mathrm{~d} x\right)^{\frac{N(q-1)}{l}}+\left(\int_{D}\left|\nabla u^{\frac{l}{2}}\right|^{2} \mathrm{~d} x\right)^{\frac{N(q-1)}{l}}\right) \\
& =C^{\frac{2 N(q-1)}{l}}\left(\left(\int_{D} u^{l} \mathrm{~d} x\right)^{1+\frac{2(q-1)}{l}}+\left(\int_{D} u^{l} \mathrm{~d} x\right)^{1-\frac{(N-2)(q-1)}{l}}\left(\int_{D}\left|\nabla u^{\frac{l}{2}}\right|^{2} \mathrm{~d} x\right)^{\frac{N(q-1)}{l}}\right) \\
& =C^{\frac{2 N(q-1)}{l}}\left(\left(\int_{D} u^{l} \mathrm{~d} x\right)^{1+\frac{2(q-1)}{l}}\right. \\
& \left.+\left(\varepsilon_{2}^{\frac{N(1-q)}{l-N(q-1)}}\left(\int_{D} u^{l} \mathrm{~d} x\right)^{1+\frac{2(q-1)}{l-N(q-1)}}\right)^{1-\frac{N(q-1)}{l}}\left(\varepsilon_{2} \int_{D}\left|\nabla u^{\frac{l}{2}}\right|^{2} \mathrm{~d} x\right)^{\frac{N(q-1)}{l}}\right) \\
& \leq C^{\frac{2 N(q-1)}{l}}\left(\left(\int_{D} u^{l} \mathrm{~d} x\right)^{1+\frac{2(q-1)}{l}}+\left(1-\frac{N(q-1)}{l}\right) \varepsilon^{\frac{N(1-q)}{l-N(q-1)}}\left(\int_{D} u^{l} \mathrm{~d} x\right)^{1+\frac{2(q-1)}{l-N(q-1)}}\right. \\
& \left.+\frac{N(q-1)}{l} \varepsilon_{2} \int_{D}\left|\nabla u^{\frac{l}{2}}\right|^{2} \mathrm{~d} x\right)
\end{aligned}
$$

where $\varepsilon_{2}=\frac{\theta(l-1)}{I_{1} N(q-1)}, I_{1}=l \sigma C^{\frac{2 N(q-1)}{l}}\left(\frac{N^{2}}{2 \rho_{0}^{2}}+\frac{(l+q-1)^{2} d^{2}}{2 \varepsilon_{1} \rho_{0}^{2}}\right)$.

Then we deal with the fourth term of (4.14). Due to (4.6), we get $0<\frac{(m-1)(N-2)}{2 l}<1$, $0<\frac{N(m-1)}{2 l}<1$. Using the Hölder inequality, the Young inequality, and (4.3)-(4.4), we de- 
rive

$$
\begin{aligned}
& \int_{D} u^{l+m-1} \mathrm{~d} x\left(\int_{D} u^{l} \mathrm{~d} x\right)^{p} \\
& \leq\left(\int_{D} u^{l} \mathrm{~d} x\right)^{1-\frac{(m-1)(N-2)}{2 l}}\left(\int_{D}\left(u^{\frac{l}{2}}\right)^{\frac{2 N}{N-2}} \mathrm{~d} x\right)^{\frac{(m-1)(N-2)}{2 l}}\left(\int_{D} u^{l} \mathrm{~d} x\right)^{p} \\
& =\left(\int_{D} u^{l} \mathrm{~d} x\right)^{1+p-\frac{(m-1)(N-2)}{2 l}}\left(\left(\int_{D}\left(u^{\frac{l}{2}}\right)^{\frac{2 N}{N-2}} \mathrm{~d} x\right)^{\frac{N-2}{2 N}}\right)^{\frac{N(m-1)}{l}} \\
& \leq\left(\int_{D} u^{l} \mathrm{~d} x\right)^{1+p-\frac{(m-1)(N-2)}{2 l}}\left(C\left(\int_{D} u^{l} \mathrm{~d} x+\int_{D}\left|\nabla u^{\frac{l}{2}}\right|^{2} \mathrm{~d} x\right)^{\frac{1}{2}}\right)^{\frac{N(m-1)}{l}} \\
& =C^{\frac{N(m-1)}{l}}\left(\int_{D} u^{l} \mathrm{~d} x\right)^{1+p-\frac{(m-1)(N-2)}{2 l}}\left(\int_{D} u^{l} \mathrm{~d} x+\int_{D}\left|\nabla u^{\frac{l}{2}}\right|^{2} \mathrm{~d} x\right)^{\frac{N(m-1)}{2 l}} \\
& \leq C^{\frac{N(m-1)}{l}}\left(\int_{D} u^{l} \mathrm{~d} x\right)^{1+p-\frac{(m-1)(N-2)}{2 l}}\left(\left(\int_{D} u^{l} \mathrm{~d} x\right)^{\frac{N(m-1)}{2 l}}+\left(\int_{D}\left|\nabla u^{\frac{l}{2}}\right|^{2} \mathrm{~d} x\right)^{\frac{N(m-1)}{2 l}}\right) \\
& =C^{\frac{N(m-1)}{l}}\left(\left(\int_{D} u^{l} \mathrm{~d} x\right)^{1+p+\frac{m-1}{l}}+\left(\int_{D} u^{l} \mathrm{~d} x\right)^{1+p-\frac{(m-1)(N-2)}{2 l}}\left(\int_{D}\left|\nabla u^{\frac{l}{2}}\right|^{2} \mathrm{~d} x\right)^{\frac{N(m-1)}{2 l}}\right) \\
& =C^{\frac{N(m-1)}{l}}\left(\left(\varepsilon_{3}\left(\int_{D} u^{l} \mathrm{~d} x\right)^{\frac{l+r-1}{l}}\right)^{\frac{l}{l+r-1}}\left(\varepsilon_{3}^{\frac{l}{1-r}}\left(\int_{D} u^{l} \mathrm{~d} x\right)^{\frac{(l p+m-1)(l+r-1)}{l(r-1)}}\right)^{\frac{r-1}{l+r-1}}\right. \\
& \left.+\left(\varepsilon_{4}^{\frac{N(1-m)}{2 l-N(m-1)}}\left(\int_{D} u^{l} \mathrm{~d} x\right)^{1+\frac{2 p l+2(m-1)}{2 l-N(m-1)}}\right)^{1-\frac{N(m-1)}{2 l}}\left(\varepsilon_{4} \int_{D}\left|\nabla u^{\frac{l}{2}}\right|^{2} \mathrm{~d} x\right)^{\frac{N(m-1)}{2 l}}\right) \\
& \leq C^{\frac{N(m-1)}{l}}\left(\frac{l}{l+r-1} \varepsilon_{3}\left(\int_{D} u^{l} \mathrm{~d} x\right)^{\frac{l+r-1}{l}}+\frac{r-1}{l+r-1} \varepsilon_{3}^{\frac{l}{1-r}}\left(\int_{D} u^{l} \mathrm{~d} x\right)^{\frac{(l p+m-1)(l+r-1)}{l(r-1)}}\right. \\
& +\left(1-\frac{N(m-1)}{2 l}\right) \varepsilon_{4}^{\frac{N(1-m)}{2 l-N(m-1)}}\left(\int_{D} u^{l} \mathrm{~d} x\right)^{1+\frac{2 p l+2(m-1)}{2 l-N(m-1)}} \\
& \left.+\frac{N(m-1)}{2 l} \varepsilon_{4} \int_{D}\left|\nabla u^{\frac{l}{2}}\right|^{2} \mathrm{~d} x\right),
\end{aligned}
$$

where $\varepsilon_{3}=\frac{\gamma_{2}(l+r-1)}{l \gamma_{1}} C^{\frac{N(1-m)}{l}}|D|^{\frac{1-r}{l}}, \varepsilon_{4}=\frac{2 \theta(l-1)}{l \gamma_{1} N(m-1)} C^{\frac{N(1-m)}{l}}$.

Finally we compute the last term of (4.14). Applying the Hölder inequality, we deduce

$$
\int_{D} u^{l} \mathrm{~d} x \leq|D|^{\frac{r-1}{l+r-1}}\left(\int_{D} u^{l+r-1} \mathrm{~d} x\right)^{\frac{l}{l+r-1}}
$$

that is,

$$
\int_{D} u^{l+r-1} \mathrm{~d} x \geq|D|^{\frac{1-r}{l}}\left(\int_{D} u^{l} \mathrm{~d} x\right)^{\frac{l+r-1}{l}} .
$$

Substituting (4.15)-(4.17) into (4.14), we have

$$
\begin{aligned}
A^{\prime}(t) \leq & \frac{l \sigma}{2} \int_{D} u^{l} \mathrm{~d} x+I_{1}\left(\int_{D} u^{l} \mathrm{~d} x\right)^{1+\frac{2(q-1)}{l}} \\
& +\frac{I_{1}[l-N(q-1)]}{l} \varepsilon_{2}^{\frac{N(1-q)}{l-N(q-1)}}\left(\int_{D} u^{l} \mathrm{~d} x\right)^{1+\frac{2(q-1)}{l-N(q-1)}}
\end{aligned}
$$




$$
\begin{aligned}
& +\frac{l \gamma_{1}(r-1)}{l+r-1} C^{\frac{N(m-1)}{l}} \varepsilon_{3}^{\frac{l}{1-r}}\left(\int_{D} u^{l} \mathrm{~d} x\right)^{\frac{(l p+m-1)(l+r-1)}{l(r-1)}} \\
& +\frac{\gamma_{1}[2 l-N(m-1)]}{2} C^{\frac{N(m-1)}{l}} \varepsilon_{4}^{\frac{N(1-m)}{2 l-N(m-1)}}\left(\int_{D} u^{l} \mathrm{~d} x\right)^{1+\frac{2 p l+2(m-1)}{2 l-N(m-1)}} .
\end{aligned}
$$

By (4.1), we obtain

$$
A(t)=l \int_{D} \int_{0}^{u} y^{l-1} g^{\prime}(y) \mathrm{d} y \mathrm{~d} x \geq \xi \int_{D} u^{l} \mathrm{~d} x
$$

which implies

$$
\int_{D} u^{l} \mathrm{~d} x \leq \frac{1}{\xi} A(t)
$$

Now, inserting (4.19) into (4.18), we derive

$$
\begin{aligned}
A^{\prime}(t) \leq & \frac{l \sigma}{2 \xi} A(t)+\frac{I_{1}}{\xi^{1+\frac{2(q-1)}{l}}} A^{1+\frac{2(q-1)}{l}}(t)+\frac{I_{1}[l-N(q-1)]}{l \xi^{1+\frac{2(q-1)}{l-N(q-1)}}} \varepsilon_{2}^{\frac{N(1-q)}{l-N(q-1)}} A^{1+\frac{2(q-1)}{l-N(q-1)}}(t) \\
& +\frac{l \gamma_{1}(r-1)}{(l+r-1) \xi^{\frac{(l p+m-1)(l+r-1)}{l(r-1)}}} C^{\frac{N(m-1)}{l}} \varepsilon_{3}^{\frac{l}{1-r}} A^{\frac{(l p+m-1)(l+r-1)}{l(r-1)}}(t) \\
& +\frac{\gamma_{1}[2 l-N(m-1)]}{2 \xi^{1+\frac{2 p l+2(m-1)}{2 l-N(m-1)}}} C^{\frac{N(m-1)}{l}} \varepsilon_{4}^{\frac{N(1-m)}{2 l-N(m-1)}} A^{1+\frac{2 p l+2(m-1)}{2 l-N(m-1)}}(t) \\
= & J_{1} A(t)+J_{2} A^{1+\frac{2(q-1)}{l}}(t)+J_{3} A^{1+\frac{2(q-1)}{l-N(q-1)}}(t) \\
& +J_{4} A^{\frac{(l p+m-1)(l+r-1)}{l(r-1)}}(t)+J_{5} A^{1+\frac{2 p l+2(m-1)}{2 l-N(m-1)}}(t),
\end{aligned}
$$

where $J_{1}, J_{2}, J_{3}, J_{4}$, and $J_{5}$ are defined in (4.7)-(4.10). Since $\lim _{t \rightarrow t^{*-}} A(t)=+\infty$, we integrate (4.20) from 0 to $t^{*}$ to get

$$
t^{*} \geq \int_{A(0)}^{+\infty} \frac{\mathrm{d} \tau}{J_{1} \tau+J_{2} \tau^{1+\frac{2(q-1)}{l}}+J_{3} \tau^{1+\frac{2(q-1)}{l-N(q-1)}}+J_{4} \tau^{\frac{(l p+m-1)(l+r-1)}{l(r-1)}}+J_{5} \tau^{1+\frac{2 p l+2(m-1)}{2 l-N(m-1)}}}
$$

\section{Lower bounds of the blow-up time $t^{*}$ in $D \subset \mathbb{R}^{2}$}

In this section, we search for a lower bound of $t^{*}$ in $D \subset \mathbb{R}^{2}$. Suppose that the functions $h$ and $g$ satisfy

$$
h(s) \leq \tilde{\sigma} s^{\tilde{q}}, \quad g^{\prime}(s) \geq \tilde{\xi}, \quad s \geq 0
$$

where $\tilde{\sigma}, \tilde{q}$, and $\tilde{\xi}$ are some positive constants and

$$
\tilde{q}>1 \text {. }
$$

By [17, Corollary 9.14], we have

$$
W^{1,2} \hookrightarrow L^{4}, \quad \text { where } N=2 \text {, }
$$


which implies the following Sobolev inequality:

$$
\left(\int_{D}\left(u^{\frac{l}{2}}\right)^{4} \mathrm{~d} x\right)^{\frac{1}{4}} \leq \tilde{C}\left(\int_{D} u^{l} \mathrm{~d} x+\int_{D}\left|\nabla u^{\frac{l}{2}}\right|^{2} \mathrm{~d} x\right)^{\frac{1}{2}}
$$

where $\tilde{C}$ is a Sobolev embedding constant depending on $D$. We need to use inequality (5.3) to get a lower bound of $t^{*}$. Now, we define auxiliary functions as follows:

$$
\tilde{A}(t)=\int_{D} \tilde{B}(u) \mathrm{d} x, \quad t \geq 0, \quad \tilde{B}(s)=l \int_{0}^{s} y^{l-1} g^{\prime}(y) \mathrm{d} y, \quad s \geq 0 .
$$

The main result of this section is the following Theorem 5.1.

Theorem 5.1 Let $u(x, t)$ be a nonnegative classical solution of $(1.1)$ in $D \subset \mathbb{R}^{2}$. Assume that (5.1)-(5.2) hold and

$$
r>1, \quad m>1, \quad l>\max \{2,2(m-1), 4(\tilde{q}-1)\} .
$$

In addition, we assume that $u(x, t)$ blows up at some finite time $t^{*}$ in the measure $\tilde{A}(t)$. Then $t^{*}$ is bounded below by

$$
t^{*} \geq \int_{\tilde{A}(0)}^{+\infty} \frac{\mathrm{d} \tau}{\tilde{J}_{1} \tau+\tilde{J}_{2} \tau^{1+\frac{2(\tilde{q}-1)}{l}}+\tilde{J}_{3} \tau^{1+\frac{2(\tilde{q}-1)}{l-4(\tilde{q}-1)}}+\tilde{J}_{4} \tau^{\frac{(l p+m-1)(l+r-1)}{l(r-1)}}+\tilde{J}_{5} \tau^{\frac{l(1+p)-(m-1)}{l-2(m-1)}}},
$$

where

$$
\begin{aligned}
& \tilde{J}_{1}=\frac{l \tilde{\sigma}}{2 \tilde{\xi}}, \quad \tilde{J}_{2}=\frac{l \tilde{\sigma}}{\rho_{0}^{2} \tilde{\xi}^{1+\frac{2(\tilde{q}-1)}{l}}} \tilde{C}^{\frac{8(\tilde{q}-1)}{l}}\left(2+\frac{\tilde{\sigma}(l+\tilde{q}-1)^{2} \tilde{d}^{2}}{2 \theta(l-1)}\right), \\
& \tilde{J}_{3}=\frac{l-4(\tilde{q}-1)}{\tilde{\xi}^{1+\frac{2(\tilde{q}-1)}{l-4(q-1)}}}\left(\frac{\theta(l-1)}{4 l(\tilde{q}-1) \tilde{C}^{2}}\right)^{\frac{4(1-\tilde{q})}{l-4(\tilde{q}-1)}}\left[\frac{\tilde{\sigma}}{\tilde{\rho}_{0}^{2}}\left(2+\frac{\tilde{\sigma}(l+\tilde{q}-1)^{2} \tilde{d}^{2}}{2 \theta(l-1)}\right)\right]^{\frac{l}{l-4(\tilde{q}-1)}}
\end{aligned}
$$

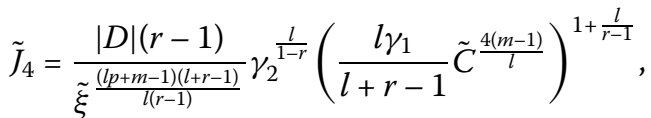

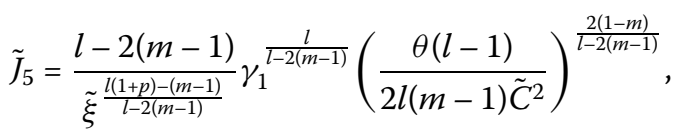

$$
\begin{aligned}
& \tilde{\rho}_{0}=\min _{x \in \partial D}(x \cdot v), \quad \tilde{d}=\max _{x \in \bar{D}}|x|,
\end{aligned}
$$

and $\theta$ is defined in (1.2).

Proof Repeating the calculation process of (4.12)-(4.14) and noting $N=2$ in this section, we get

$$
\begin{aligned}
\tilde{A}^{\prime}(t) \leq & \frac{l \tilde{\sigma}}{2} \int_{D} u^{l} \mathrm{~d} x+l \tilde{\sigma}\left(\frac{2}{\tilde{\rho}_{0}^{2}}+\frac{(l+\tilde{q}-1)^{2} \tilde{d}^{2}}{2 \tilde{\varepsilon}_{1} \tilde{\rho}_{0}^{2}}\right) \int_{D} u^{l+2 \tilde{q}-2} \mathrm{~d} x \\
& +\left(\frac{2 \tilde{\varepsilon}_{1} \tilde{\sigma}}{l}-\frac{4 \theta(l-1)}{l}\right) \int_{D}\left|\nabla u^{\frac{l}{2}}\right|^{2} \mathrm{~d} x
\end{aligned}
$$




$$
+l \gamma_{1} \int_{D} u^{l+m-1} \mathrm{~d} x\left(\int_{D} u^{l} \mathrm{~d} x\right)^{p}-l \gamma_{2} \int_{D} u^{l+r-1} \mathrm{~d} x
$$

where $\tilde{\rho}_{0}$ and $\tilde{d}$ are defined in (5.10) and $\tilde{\varepsilon}_{1}=\frac{\theta(l-1)}{\tilde{\sigma}}$.

First, we focus on the second term of (5.11). By (5.2) and (5.5), we get $0<\frac{2(\tilde{q}-1)}{l}<1$ and $0<\frac{4(\tilde{q}-1)}{l}<1$. Now we apply the Hölder inequality, the Young inequality, (4.4), and (5.3) to deduce

$$
\begin{aligned}
& \int_{D} u^{l+2 \tilde{q}-2} \mathrm{~d} x \\
& \leq\left(\int_{D} u^{l} \mathrm{~d} x\right)^{1-\frac{2(\tilde{q}-1)}{l}}\left(\int_{D}\left(u^{\frac{l}{2}}\right)^{4} \mathrm{~d} x\right)^{\frac{2(\tilde{q}-1)}{l}} \\
& =\left(\int_{D} u^{l} \mathrm{~d} x\right)^{1-\frac{2(\tilde{q}-1)}{l}}\left(\left(\int_{D}\left(u^{\frac{l}{2}}\right)^{4} \mathrm{~d} x\right)^{\frac{1}{4}}\right)^{\frac{8(\tilde{q}-1)}{l}} \\
& \leq\left(\int_{D} u^{l} \mathrm{~d} x\right)^{1-\frac{2(\tilde{q}-1)}{l}}\left(\tilde{C}\left(\int_{D} u^{l} \mathrm{~d} x+\int_{D}\left|\nabla u^{\frac{l}{2}}\right|^{2} \mathrm{~d} x\right)^{\frac{1}{2}}\right)^{\frac{8(\tilde{q}-1)}{l}} \\
& =\tilde{C}^{\frac{8(\tilde{q}-1)}{l}}\left(\int_{D} u^{l} \mathrm{~d} x\right)^{1-\frac{2(\tilde{q}-1)}{l}}\left(\int_{D} u^{l} \mathrm{~d} x+\int_{D}\left|\nabla u^{\frac{l}{2}}\right|^{2} \mathrm{~d} x\right)^{\frac{4(\tilde{q}-1)}{l}} \\
& \leq \tilde{C}^{\frac{8(\tilde{q}-1)}{l}}\left(\int_{D} u^{l} \mathrm{~d} x\right)^{1-\frac{2(\tilde{q}-1)}{l}}\left(\left(\int_{D} u^{l} \mathrm{~d} x\right)^{\frac{4(\tilde{q}-1)}{l}}+\left(\int_{D}\left|\nabla u^{\frac{l}{2}}\right|^{2} \mathrm{~d} x\right)^{\frac{4(\tilde{q}-1)}{l}}\right) \\
& =\tilde{C}^{\frac{8(\tilde{q}-1)}{l}}\left(\left(\int_{D} u^{l} \mathrm{~d} x\right)^{1+\frac{2(\tilde{q}-1)}{l}}+\left(\int_{D} u^{l} \mathrm{~d} x\right)^{1-\frac{2(\tilde{q}-1)}{l}}\left(\int_{D}\left|\nabla u^{\frac{l}{2}}\right|^{2} \mathrm{~d} x\right)^{\frac{4(\tilde{q}-1)}{l}}\right) \\
& =\tilde{C}^{\frac{8(\tilde{q}-1)}{l}}\left(\left(\int_{D} u^{l} \mathrm{~d} x\right)^{1+\frac{2(\tilde{q}-1)}{l}}\right. \\
& \left.+\left(\tilde{\varepsilon}_{2}^{\frac{4(1-\tilde{q})}{l-4(\tilde{q}-1)}}\left(\int_{D} u^{l} \mathrm{~d} x\right)^{1+\frac{2(\tilde{q}-1)}{l-4(\tilde{q}-1)}}\right)^{1-\frac{4(\tilde{q}-1)}{l}}\left(\tilde{\varepsilon}_{2} \int_{D}\left|\nabla u^{\frac{l}{2}}\right|^{2} \mathrm{~d} x\right)^{\frac{4(\tilde{q}-1)}{l}}\right) \\
& \leq \tilde{C}^{\frac{8(\tilde{q}-1)}{l}}\left(\left(\int_{D} u^{l} \mathrm{~d} x\right)^{1+\frac{2(\tilde{q}-1)}{l}}+\left(1-\frac{4(\tilde{q}-1)}{l}\right) \tilde{\varepsilon}^{\frac{4(1-\tilde{q})}{l-4(\tilde{q}-1)}}\left(\int_{D} u^{l} \mathrm{~d} x\right)^{1+\frac{2(\tilde{q}-1)}{l-4(\tilde{q}-1)}}\right. \\
& \left.+\frac{4(\tilde{q}-1)}{l} \tilde{\varepsilon}_{2} \int_{D}\left|\nabla u^{\frac{l}{2}}\right|^{2} \mathrm{~d} x\right)
\end{aligned}
$$

where $\tilde{\varepsilon}_{2}=\frac{\theta(l-1)}{4 \tilde{l}_{1}(\tilde{q}-1)}, \tilde{I}_{1}=l \tilde{\sigma} \tilde{C}^{\frac{8(\tilde{q}-1)}{l}}\left(\frac{2}{\tilde{\rho}_{0}^{2}}+\frac{\left(l+\tilde{q}_{-1}\right)^{2} \tilde{d}^{2}}{2 \tilde{\varepsilon}_{1} \tilde{\rho}_{0}^{2}}\right)$.

Next, we deal with the fourth term of (5.11). Due to (5.5), we get $0<\frac{m-1}{l}<1$, $0<\frac{2(m-1)}{l}<1$. Using the Hölder inequality, the Young inequality, (4.4), and (5.3), we derive

$$
\begin{aligned}
& \int_{D} u^{l+m-1} \mathrm{~d} x\left(\int_{D} u^{l} \mathrm{~d} x\right)^{p} \\
& \leq\left(\int_{D} u^{l} \mathrm{~d} x\right)^{1-\frac{m-1}{l}}\left(\int_{D}\left(u^{\frac{l}{2}}\right)^{4} \mathrm{~d} x\right)^{\frac{m-1}{l}}\left(\int_{D} u^{l} \mathrm{~d} x\right)^{p} \\
& =\left(\int_{D} u^{l} \mathrm{~d} x\right)^{1+p-\frac{m-1}{l}}\left(\left(\int_{D}\left(u^{\frac{l}{2}}\right)^{4} \mathrm{~d} x\right)^{\frac{1}{4}}\right)^{\frac{4(m-1)}{l}}
\end{aligned}
$$




$$
\begin{aligned}
& \leq\left(\int_{D} u^{l} \mathrm{~d} x\right)^{1+p-\frac{m-1}{l}}\left(\tilde{C}\left(\int_{D} u^{l} \mathrm{~d} x+\int_{D}\left|\nabla u^{\frac{l}{2}}\right|^{2} \mathrm{~d} x\right)^{\frac{1}{2}}\right)^{\frac{4(m-1)}{l}} \\
& =\tilde{C}^{\frac{4(m-1)}{l}}\left(\int_{D} u^{l} \mathrm{~d} x\right)^{1+p-\frac{m-1}{l}}\left(\int_{D} u^{l} \mathrm{~d} x+\int_{D}\left|\nabla u^{\frac{l}{2}}\right|^{2} \mathrm{~d} x\right)^{\frac{2(m-1)}{l}} \\
& \leq \tilde{C}^{\frac{4(m-1)}{l}}\left(\int_{D} u^{l} \mathrm{~d} x\right)^{1+p-\frac{m-1}{l}}\left(\left(\int_{D} u^{l} \mathrm{~d} x\right)^{\frac{2(m-1)}{l}}+\left(\int_{D}\left|\nabla u^{\frac{l}{2}}\right|^{2} \mathrm{~d} x\right)^{\frac{2(m-1)}{l}}\right) \\
& =\tilde{C}^{\frac{4(m-1)}{l}}\left(\left(\int_{D} u^{l} \mathrm{~d} x\right)^{1+p+\frac{m-1}{l}}+\left(\int_{D} u^{l} \mathrm{~d} x\right)^{1+p-\frac{m-1}{l}}\left(\int_{D}\left|\nabla u^{\frac{l}{2}}\right|^{2} \mathrm{~d} x\right)^{\frac{2(m-1)}{l}}\right) \\
& =\tilde{C}^{\frac{4(m-1)}{l}}\left(\left(\tilde{\varepsilon}_{3}\left(\int_{D} u^{l} \mathrm{~d} x\right)^{\frac{l+r-1}{l}}\right)^{\frac{l}{l+r-1}}\left(\tilde{\varepsilon}_{3}^{\frac{l}{1-r}}\left(\int_{D} u^{l} \mathrm{~d} x\right)^{\frac{(l p+m-1)(l+r-1)}{l(r-1)}}\right)^{\frac{r-1}{l+r-1}}\right. \\
& \left.+\left(\tilde{\varepsilon}_{4}^{\frac{2(1-m)}{l-2(m-1)}}\left(\int_{D} u^{l} \mathrm{~d} x\right)^{\frac{l(1+p)-(m-1)}{l-2(m-1)}}\right)^{1-\frac{2(m-1)}{l}}\left(\tilde{\varepsilon}_{4} \int_{D}\left|\nabla u^{\frac{l}{2}}\right|^{2} \mathrm{~d} x\right)^{\frac{2(m-1)}{l}}\right) \\
& \leq \tilde{C}^{\frac{4(m-1)}{l}}\left(\frac{l}{l+r-1} \tilde{\varepsilon}_{3}\left(\int_{D} u^{l} \mathrm{~d} x\right)^{\frac{l+r-1}{l}}+\frac{r-1}{l+r-1} \tilde{\varepsilon}_{3}^{\frac{l}{1-r}}\left(\int_{D} u^{l} \mathrm{~d} x\right)^{\frac{(l p+m-1)(l+r-1)}{l(r-1)}}\right. \\
& +\left(1-\frac{2(m-1)}{l}\right) \tilde{\varepsilon}^{\frac{2(1-m)}{l-2(m-1)}}\left(\int_{D} u^{l} \mathrm{~d} x\right)^{\frac{l(1+p)-(m-1)}{l-2(m-1)}} \\
& \left.+\frac{2(m-1)}{l} \tilde{\varepsilon}_{4} \int_{D}\left|\nabla u^{\frac{l}{2}}\right|^{2} \mathrm{~d} x\right)
\end{aligned}
$$

where $\tilde{\varepsilon}_{3}=\frac{\gamma_{2}(l+r-1)}{l \gamma_{1}} \tilde{C}^{\frac{4(1-m)}{l}}|D|^{\frac{1-r}{l}}, \tilde{\varepsilon}_{4}=\frac{\theta(l-1)}{2 l \gamma_{1}(m-1)} \tilde{C}^{\frac{4(1-m)}{l}}$.

Finally, we consider the last term of (5.11). Repeating the calculation process of (4.17), we have

$$
\int_{D} u^{l+r-1} \mathrm{~d} x \geq|D|^{\frac{1-r}{l}}\left(\int_{D} u^{l} \mathrm{~d} x\right)^{\frac{l+r-1}{l}} .
$$

Substituting (5.12)-(5.14) into (5.11), we deduce

$$
\begin{aligned}
\tilde{A}^{\prime}(t) \leq & \frac{l \tilde{\sigma}}{2} \int_{D} u^{l} \mathrm{~d} x+\tilde{I}_{1}\left(\int_{D} u^{l} \mathrm{~d} x\right)^{1+\frac{2(\tilde{q}-1)}{l}} \\
& +\frac{\tilde{I}_{1}[l-4(\tilde{q}-1)]}{l} \tilde{\varepsilon}_{2}^{\frac{4(1-\tilde{q})}{l(\tilde{q}-1)}}\left(\int_{D} u^{l} \mathrm{~d} x\right)^{1+\frac{2(\tilde{q}-1)}{l-4(\tilde{q}-1)}} \\
& +\frac{l \gamma_{1}(r-1)}{l+r-1} \tilde{C}^{\frac{4(m-1)}{l}} \tilde{\varepsilon}_{3}^{\frac{l}{l-r}}\left(\int_{D} u^{l} \mathrm{~d} x\right)^{\frac{(l p+m-1)(l+r-1)}{l(r-1)}} \\
& +\gamma_{1}[l-2(m-1)] \tilde{C}^{\frac{4(m-1)}{l}} \tilde{\varepsilon}_{4}^{\frac{2(1-m)}{l-2(m-1)}}\left(\int_{D} u^{l} \mathrm{~d} x\right)^{\frac{l(1+p)-(m-1)}{l-2(m-1)}} .
\end{aligned}
$$

We repeat the calculation process of (4.19) to obtain

$$
\int_{D} u^{l} \mathrm{~d} x \leq \frac{1}{\tilde{\xi}} \tilde{A}(t)
$$


Now, inserting (5.16) into (5.15), we derive

$$
\begin{aligned}
\tilde{A}^{\prime}(t) \leq & \frac{l \tilde{\sigma}}{2 \tilde{\xi}} \tilde{A}(t)+\frac{\tilde{I}_{1}}{\tilde{\xi}^{1+\frac{2(\tilde{q}-1)}{l}}} \tilde{A}^{1+\frac{2(\tilde{q}-1)}{l}}(t)+\frac{\tilde{I}_{1}[l-4(\tilde{q}-1)]}{l \tilde{\xi}^{1+\frac{2(\tilde{q}-1)}{l-4(\tilde{q}-1)}}} \tilde{\varepsilon}_{2}^{\frac{4(1-\tilde{q})}{l-4(\tilde{q}-1)}} \tilde{A}^{1+\frac{2(\tilde{q}-1)}{l-4(\tilde{q}-1)}}(t) \\
& +\frac{l \gamma_{1}(r-1)}{(l+r-1) \tilde{\xi}^{\frac{(l p+m-1)(l+r-1)}{l(r-1)}}} \tilde{C}^{\frac{4(m-1)}{l}} \tilde{\varepsilon}_{3}^{\frac{l}{1-r}} \tilde{A}^{\frac{(l p+m-1)(l+r-1)}{l(r-1)}}(t) \\
& +\frac{\gamma_{1}[l-2(m-1)]}{\tilde{\xi}^{\frac{l(1+p)-(m-1)}{l-2(m-1)}}} \tilde{C}^{\frac{4(m-1)}{l}} \tilde{\varepsilon}_{4}^{\frac{2(1-m)}{l-2(m-1)}} \tilde{A}^{\frac{l(1+p)-(m-1)}{l-2(m-1)}}(t) \\
= & \tilde{J}_{1} \tilde{A}(t)+\tilde{J}_{2} \tilde{A}^{1+\frac{2(\tilde{q}-1)}{l}}(t)+\tilde{J}_{3} \tilde{A}^{1+\frac{2(\tilde{q}-1)}{l-4(\tilde{q}-1)}}(t) \\
& +\tilde{J}_{4} \tilde{A}^{\frac{(l p+m-1)(l+r-1)}{l(r-1)}}(t)+\tilde{J}_{5} \tilde{A}^{\frac{l(1+p)-(m-1)}{l-2(m-1)}}(t)
\end{aligned}
$$

where $\tilde{J}_{1}, \tilde{J}_{2}, \tilde{J}_{3}, \tilde{J}_{4}$, and $\tilde{J}_{5}$ are defined in (5.6)-(5.9). Since $\lim _{t \rightarrow t^{*-}} \tilde{A}(t)=+\infty$, we integrate (5.17) from 0 to $t^{*}$ to get

$$
t^{*} \geq \int_{\tilde{A}(0)}^{+\infty} \frac{\mathrm{d} \tau}{\tilde{J}_{1} \tau+\tilde{J}_{2} \tau^{1+\frac{2(\tilde{q}-1)}{l}}+\tilde{J}_{3} \tau^{1+\frac{2(\tilde{q}-1)}{l-4(\tilde{q}-1)}}+\tilde{J}_{4} \tau^{\frac{(l p+m-1)(l+r-1)}{l(r-1)}}+\tilde{J}_{5} \tau^{\frac{l(1+p)-(m-1)}{l-2(m-1)}}} .
$$

\section{Applications}

In what follows, three examples are given to demonstrate the results of Theorems 2.1-5.1 obtained in this paper.

Example 6.1 Let $u(x, t)$ be a nonnegative classical solution of the following problem:

$$
\begin{cases}((u+\ln (1+u)))_{t}=\sum_{i=1}^{3}\left(\left(1+|x|^{2}\right) u_{x_{i}}\right)_{x_{i}}+u^{\frac{1}{2}}\left(\int_{D} u^{3} \mathrm{~d} x\right)^{\frac{1}{3}}-u^{\frac{5}{2}} & \text { in } D \times\left(0, t^{*}\right) \\ \sum_{i=1}^{3}\left(1+|x|^{2}\right) u_{x_{i}} v_{i}=\frac{2 \sqrt{5}}{5} u^{\frac{3}{2}} & \text { on } \partial D \times\left(0, t^{*}\right) \\ u(x, 0)=u_{0}(x)=1+|x|^{2} & \text { in } \bar{D},\end{cases}
$$

where $D=\left\{x=\left.\left(x_{1}, x_{2}, x_{3}\right)|| x\right|^{2}=\sum_{i=1}^{3} x_{i}^{2}<\frac{1}{4}\right\}$ is a ball of $\mathbb{R}^{3}$. Here,

$$
\begin{aligned}
& g(u)=u+\ln (1+u), \quad h(u)=\frac{2 \sqrt{5}}{5} u^{\frac{3}{2}}, \\
& \left(a^{i j}(x)\right)_{3 \times 3}=\left(\begin{array}{ccc}
1+|x|^{2} & 0 & 0 \\
0 & 1+|x|^{2} & 0 \\
0 & 0 & 1+|x|^{2}
\end{array}\right), \quad u_{0}(x)=1+|x|^{2} .
\end{aligned}
$$

Now, $N=3, \gamma_{1}=1, \gamma_{2}=1, m=\frac{1}{2}, l=3, p=\frac{1}{3}$, and $r=\frac{5}{2}$. Choosing $\zeta=2, \gamma=\frac{2 \sqrt{5}}{5}$, and $q=\frac{3}{2}$, we can verify that (2.2)-(2.3) are satisfied. Hence, Theorem 2.1 implies that $u$ exists for all time in $\Phi(t)$ with

$$
\Phi(t)=\int_{D} G(u) \mathrm{d} x=\int_{D}\left(u^{3}+\frac{3}{2} u^{2}-3 u+3 \ln (1+u)\right) \mathrm{d} x
$$


Example 6.2 Let $u(x, t)$ be a nonnegative classical solution of the following problem:

$$
\begin{cases}\left(\frac{u+\ln (1+u)}{2}\right)_{t}=\sum_{i=1}^{3}\left(\left(1+|x|^{2}\right) u_{x_{i}}\right)_{x_{i}}+\frac{9}{4} u^{\frac{3}{2}}\left(\int_{D} u^{3} \mathrm{~d} x\right)^{\frac{1}{10}}-\frac{7}{4} u^{\frac{7}{5}} & \text { in } D \times\left(0, t^{*}\right), \\ \sum_{i=1}^{3}\left(1+|x|^{2}\right) u_{x_{i}} v_{i}=\frac{3 \sqrt{5}}{5} u^{\frac{5}{4}} & \text { on } \partial D \times\left(0, t^{*}\right), \\ u(x, 0)=u_{0}(x)=1+|x|^{2} & \text { in } \bar{D},\end{cases}
$$

where $D=\left\{x=\left.\left(x_{1}, x_{2}, x_{3}\right)|| x\right|^{2}=\sum_{i=1}^{3} x_{i}^{2} .<\frac{9}{16}\right\}$ is a ball of $\mathbb{R}^{3}$. Now we have

$$
\begin{aligned}
& g(u)=\frac{u+\ln (1+u)}{2}, \quad h(u)=\frac{3 \sqrt{5}}{5} u^{\frac{5}{4}}, \\
& \left(a^{i j}(x)\right)_{3 \times 3}=\left(\begin{array}{ccc}
1+|x|^{2} & 0 & 0 \\
0 & 1+|x|^{2} & 0 \\
0 & 0 & 1+|x|^{2}
\end{array}\right), \quad u_{0}(x)=1+|x|^{2} .
\end{aligned}
$$

Here, $N=3, \gamma_{1}=\frac{9}{4}, \gamma_{2}=\frac{7}{4}, m=\frac{3}{2}, l=3, p=\frac{1}{10}, r=\frac{7}{5}$, and $|D|=\frac{9 \pi}{16}$. Now, we compute

$$
\begin{aligned}
& \Psi(t)=\int_{D} g(u) \mathrm{d} x=\frac{1}{2} \int_{D}(u+\ln (1+u)) \mathrm{d} x \\
& \Psi(0)=\frac{1}{2} \int_{D}\left(u_{0}+\ln \left(1+u_{0}\right)\right) \mathrm{d} x=1.9302 .
\end{aligned}
$$

It follows from (3.4) that $\delta=0.7436, M_{1}=0.7552$, and $M_{2}=1.0969$. It is easy to check that (3.2)-(3.3) hold. By Theorem 3.1, we deduce that $u$ must blow up in the measure $\Psi(t)$ at some finite time $t^{*}$ and

$$
t^{*} \leq \int_{\Psi(0)}^{+\infty} \frac{d \tau}{M_{1} \tau^{m+l p}-M_{2}}=\int_{1.9302}^{+\infty} \frac{d \tau}{0.7552 \tau^{\frac{9}{5}}-1.0969}=1.2304
$$

We note that (6.1) gives an upper bound of $t^{*}$. Next, we use Theorem 4.1 to obtain a lower bound of $t^{*}$. Now we select $q=\frac{5}{4}, \sigma=\frac{3 \sqrt{5}}{5}, \xi=\frac{1}{2}$, and $\theta=1$. By (4.11), we derive $\rho_{0}=d=\frac{3}{4}$. Noting $N=3$, we can verify that (4.1)-(4.2) and (4.6) hold. Moreover, according to Theorems 2.1 and 3.2 in [18], we obtain the Sobolev embedding constant $C=7.5931$. Substituting above constants into (4.7)-(4.10), it is easy to get $J_{1}=4.0249, J_{2}=287.4, J_{3}=$ $814.14, J_{4}=95979$, and $J_{5}=68.205$. By (3.6), we obtain

$$
\begin{aligned}
& B(u)=l \int_{0}^{u} y^{l-1} g^{\prime}(y) \mathrm{d} y=\frac{3}{2} \int_{0}^{u} y^{2}\left(1+\frac{1}{1+y}\right) \mathrm{d} y=\frac{1}{2} u^{3}+\frac{3}{4} u^{2}-\frac{3}{2} u+\frac{3}{2} \ln (1+u), \\
& A(t)=\int_{D} B(u) \mathrm{d} x=\int_{D}\left(\frac{1}{2} u^{3}+\frac{3}{4} u^{2}-\frac{3}{2} u+\frac{3}{2} \ln (1+u)\right) \mathrm{d} x,
\end{aligned}
$$

and

$$
A(0)=\int_{D}\left(\frac{1}{2} u_{0}^{3}+\frac{3}{4} u_{0}^{2}-\frac{3}{2} u_{0}+\frac{3}{2} \ln \left(1+u_{0}\right)\right) \mathrm{d} x=3.2897
$$

Blow-up of $u$ in the measure $\Psi(t)$ at $t^{*}$ means that $u$ blows up at $t^{*}$. Hence $u$ must also blow up in the measure $A(t)$ at $t^{*}$. By Theorem 4.1, we get a lower bound of the blow-up 
time $t^{*}$ as follows:

$$
\begin{aligned}
t^{*} & \geq T_{2}=\int_{A(0)}^{+\infty} \frac{\mathrm{d} \tau}{J_{1} \tau+J_{2} \tau^{1+\frac{2(q-1)}{l}}+J_{3} \tau^{1+\frac{2(q-1)}{l-n(q-1)}}+J_{4} \tau^{1+p+\frac{m-1}{l}}+J_{5} \tau^{1+\frac{2 p l+2(m-1)}{2 l-n(m-1)}}} \\
& =\int_{3.2897}^{+\infty} \frac{\mathrm{d} \tau}{4.0249 \tau+287.4 \tau^{\frac{7}{6}}+814.14 \tau^{\frac{11}{9}}+95979 \tau^{\frac{34}{15}}+68.205 \tau^{\frac{61}{45}}} \\
& =1.8166 \times 10^{-6} .
\end{aligned}
$$

Combining (6.1) and (6.2), we have

$$
1.8166 \times 10^{-6} \leq t^{*} \leq 1.2304
$$

Example 6.3 Let $u(x, t)$ be a nonnegative classical solution of the following problem:

$$
\begin{cases}u_{t}=\sum_{i=1}^{2}\left(\left(1+|x|^{2}\right) u_{x_{i}}\right)_{x_{i}}+2 u^{\frac{3}{2}}\left(\int_{D} u^{3} \mathrm{~d} x\right)^{\frac{1}{10}}-2 u^{\frac{7}{5}} & \text { in } D \times\left(0, t^{*}\right), \\ \sum_{i=1}^{2}\left(1+|x|^{2}\right) u_{x_{i}} v_{i}=\frac{3 \sqrt{5}}{5} u^{\frac{5}{4}} & \text { on } \partial D \times\left(0, t^{*}\right) \\ u(x, 0)=u_{0}(x)=1+|x|^{2} & \text { in } \bar{D},\end{cases}
$$

where $D=\left\{x=\left.\left(x_{1}, x_{2}\right)|| x\right|^{2}=\sum_{i=1}^{2} x_{i}^{2}<\frac{9}{16}\right\}$ is a circular region of $\mathbb{R}^{2}$. Now

$$
\begin{aligned}
& g(u)=u, \quad h(u)=\frac{3 \sqrt{5}}{5} u^{\frac{5}{4}}, \\
& \left(a^{i j}(x)\right)_{2 \times 2}=\left(\begin{array}{cc}
1+|x|^{2} & 0 \\
0 & 1+|x|^{2}
\end{array}\right), \quad u_{0}(x)=1+|x|^{2} .
\end{aligned}
$$

Here, $N=2, \gamma_{1}=2, \gamma_{2}=2, m=\frac{3}{2}, l=3, p=\frac{1}{10}, r=\frac{7}{5}$, and $|D|=\frac{9 \pi}{16}$. By (3.1), we have

$$
\Psi(t)=\int_{D} g(u) \mathrm{d} x=\int_{D} u \mathrm{~d} x, \quad \Psi(0)=\int_{D} u_{0} \mathrm{~d} x=\int_{D}\left(1+|x|^{2}\right) \mathrm{d} x=2.2642 .
$$

By (3.4), we obtain $\delta=0.6506, M_{1}=0.7552$, and $M_{2}=2.0004$. After some simple calculations, we know that (3.2)-(3.3) hold. From Theorem 3.1, it follows that $u$ blows up in the measure $\Psi(t)$ at $t^{*}$ and

$$
t^{*} \leq \int_{\Psi(0)}^{+\infty} \frac{d \tau}{M_{1} \tau^{m+l p}-M_{2}}=\int_{2.2642}^{+\infty} \frac{d \tau}{0.7552 \tau^{\frac{9}{5}}-2.0004}=1.1291
$$

Next, we apply Theorem 5.1 to get a lower bound of $t^{*}$. Now we choose $\tilde{q}=\frac{5}{4}, \tilde{\sigma}=\frac{3 \sqrt{5}}{5}$, $\tilde{\xi}=1$, and $\theta=1$. It follows from (5.10) that $\tilde{\rho}_{0}=\tilde{d}=\frac{3}{4}$. Noting $N=2$, it is easy to verify that (5.1) - (5.2) and (5.5) hold. Moreover, using Theorems 2.1 and 3.2 in [18], we derive the Sobolev embedding constant $\tilde{C}=10.887$. Inserting the above constants into (5.6)-(5.9), we deduce $\tilde{J}_{1}=2.0125, \tilde{J}_{2}=140.34, \tilde{J}_{3}=783.76, \tilde{J}_{4}=997648$, and $\tilde{J}_{5}=90$. By (5.4), we have

$$
\tilde{B}(u)=l \int_{0}^{u} y^{l-1} g^{\prime}(y) \mathrm{d} y=3 \int_{0}^{u} y^{2} \mathrm{~d} y=u^{3}, \quad \tilde{A}(t)=\int_{D} \tilde{B}(u) \mathrm{d} x=\int_{D} u^{3} \mathrm{~d} x,
$$


and

$$
\tilde{A}(0)=\int_{D} u_{0}^{3} \mathrm{~d} x=\int_{D}\left(1+|x|^{2}\right)^{3} \mathrm{~d} x=3.8959
$$

Since $u$ blows up in the measure $\Psi(t)$ at $t^{*}, u$ must blow up in the measure $\tilde{A}(t)$ at $t^{*}$. By Theorem 5.1, we get

$$
\begin{aligned}
t^{*} & \geq \int_{\tilde{A}(0)}^{+\infty} \frac{\mathrm{d} \tau}{\tilde{J}_{1} \tau+\tilde{J}_{2} \tau^{1+\frac{2(\tilde{q}-1)}{l}}+\tilde{J}_{3} \tau^{1+\frac{2(\tilde{q}-1)}{l-4(\tilde{q}-1)}}+\tilde{J}_{4} \tau^{\frac{(l p+m-1)(l+r-1)}{l(r-1)}}+\tilde{J}_{5} \tau^{\frac{l(1+p)-(m-1)}{l-2(m-1)}}} \\
& =\int_{3.8959}^{+\infty} \frac{\mathrm{d} \tau}{2.0125 \tau+140.34 \tau^{\frac{7}{6}}+783.76 \tau^{\frac{5}{4}}+997648 \tau^{\frac{34}{15}}+90 \tau^{\frac{7}{5}}} \\
& =1.4132 \times 10^{-7} .
\end{aligned}
$$

From (6.3) and (6.4), it follows that

$$
1.4132 \times 10^{-7} \leq t^{*} \leq 1.1291
$$

\section{Conclusion}

In this paper, we derive the global existence and bounds for the blow-up time of nonlinear parabolic problem (1.1) with nonlocal source. To deal with nonlocal source, we must establish some new auxiliary functions different from those in [1,2] and [11]. Furthermore, to obtain the lower bound of the blow-up time in $D \subset \mathbb{R}^{N}(N \geq 3)$ and $D \subset \mathbb{R}^{2}$, we need to use the embedding theorems in Sobolev spaces $W^{1,2} \hookrightarrow L^{\frac{2 N}{N-2}}, N \geq 3$ and $W^{1,2} \hookrightarrow L^{4}, N=2$, respectively. Applying these auxiliary functions, the embedding theorems in Sobolev spaces, and the differential inequality technique, we complete our study with the blow-up and global solutions of problem (1.1).

Acknowledgements

The authors are sincerely grateful to the editors and anonymous referees for careful reading of the original manuscript and useful comments.

Funding

This work was supported by the National Natural Science Foundation of China (No. 61473180).

Abbreviations

Not applicable.

Availability of data and materials

Not applicable.

Competing interests

The authors declare that there is no conflict of interests regarding the publication of this paper.

Authors' contributions

The authors contributed equally to the manuscript. The authors read and approved the final manuscript.

Publisher's Note

Springer Nature remains neutral with regard to jurisdictional claims in published maps and institutional affiliations.

Received: 23 October 2019 Accepted: 18 February 2020 Published online: 27 February 2020 


\section{References}

1. Li, F.S., Li, J.L.: Global existence and blow-up phenomena for nonlinear divergence form parabolic equations with inhomogeneous Neumann boundary conditions. J. Math. Anal. Appl. 385, 1005-1014 (2012)

2. Zhang, J.Z., Li, F.S.: Global existence and blow-up phenomena for divergence form parabolic equation with time-dependent coefficient in multidimensional space. Z. Angew. Math. Phys. 70, 1-16 (2019)

3. Ding, J.T., Kou, W.: Blow-up solutions for reaction diffusion equations with nonlocal boundary conditions. J. Math. Anal. Appl. 470, 1-15 (2019)

4. Ding, J.T., Shen, X.H.: Blow-up time estimates in porous medium equations with nonlinear boundary conditions. Z. Angew. Math. Phys. 69, 1-13 (2018)

5. Payne, L.E., Philippin, G.A., Vernier Piro, S.: Blow-up phenomena for a semilinear heat equation with nonlinear boundary condition, I. Z. Angew. Math. Phys. 61, 999-1007 (2010)

6. Payne, L.E., Philippin, G.A., Vernier Piro, S.: Blow-up phenomena for a semilinear heat equation with nonlinear boundary condition, II. Nonlinear Anal., Real World Appl. 73, 971-978 (2010)

7. Payne, L.E., Schaefer, P.W.: Bounds for blow-up time for the heat equation under nonlinear boundary conditions. Proc. R. Soc. Edinb., Sect. A 139, 1289-1296 (2009)

8. Ding, J.T., Shen, X.H.: Blow-up analysis in quasilinear reaction-diffusion problems with weighted nonlocal source. Comput. Math. Appl. 75, 1288-1301 (2018)

9. Jiang, L.J., Xu, Y.P.: Uniform blow-up rate for parabolic equations with a weighted nonlocal nonlinear source. J. Math. Anal. Appl. 365, 50-59 (2010)

10. Ling, Z., Lin, Z.G., Pedersen, M.: Global existence and blowup for a parabolic equation with a non-local source and absorption. Acta Appl. Math. 124, 171-186 (2013)

11. Baghaei, K., Hesaaraki, M.: Lower bounds for the blow-up time in the higher-dimensional nonlinear divergence form parabolic equations. C. R. Acad. Sci. Paris, Ser. I 351, 731-735 (2013)

12. Baghaei, K., Ghaemi, M.B., Hesaaraki, M.: Lower bounds for the blow-up time in a semilinear parabolic problem involving a variable source. Appl. Math. Lett. 27, 49-52 (2014)

13. Enache, C.: Lower bounds for blow-up time in some non-linear parabolic problems under Neumann boundary conditions. Glasg. Math. J. 53, 569-575 (2011)

14. Marras, M., Vernier Piro, S.: On global existence and bounds for blow-up time in nonlinear parabolic problems with time dependent coefficients. Discrete Contin. Dyn. Syst. 2013(Suppl.), 535-544 (2013)

15. Marras, M., Vernier Piro, S., Viglialoro, G.: Blow-up phenomena for nonlinear pseudo-parabolic equations with gradient term. Discrete Contin. Dyn. Syst., Ser. B 22, 2291-2300 (2017)

16. Zhang, L.L., Wang, H., Wang, X.Q.: Global and blow-up analysis for a class of nonlinear reaction diffusion model with Dirichlet boundary conditions. Math. Methods Appl. Sci. 17, 7789-7803 (2018)

17. Brezis, H.: Functional Analysis, Sobolev Spaces and Partial Differential Equations. Universitext. Springer, New York (2011)

18. Mizuguchi, M., Tanaka, K., Sekine, K., Oishi, S.: Estimation of Sobolev embedding constant on a domain dividable into bounded convex domains. J. Inequal. Appl. 2017, 299 (2017)

\section{Submit your manuscript to a SpringerOpen ${ }^{\circ}$ journal and benefit from:}

- Convenient online submission

- Rigorous peer review

- Open access: articles freely available online

- High visibility within the field

Retaining the copyright to your article

Submit your next manuscript at $\boldsymbol{~ s p r i n g e r o p e n . c o m ~}$ 